\title{
Fungos Anamorfos (hyphomycetes) da Floresta Nacional de Caxiuanã, Pará, Brasil. Novos registros para o Neotrópico ${ }^{1}$
}

\author{
Josiane Santana Monteiro², Antonio Hernández Gutiérrez ${ }^{3}$ e Helen Maria Pontes Sotão 2,4
}

Recebido em 16/11/2009. Aceito em 21/06/2010

RESUMO - (Fungos Anamorfos (hyphomycetes) da Floresta Nacional de Caxiuanã, Pará, Brasil. Novos registros para o Neotrópico). Os hifomicetos são importantes decompositores e recicladores da matéria orgânica morta no ambiente e podem ser importantes patógenos de plantas e animais. Como parte do inventário da diversidade dos fungos sobre palmeiras da Amazônia Oriental, no sítio do Programa de Biodiversidade da Amazônia (PPBIO), na Floresta Nacional de Caxiuanã, Pará, foram identificados cinco novos registros de hifomicetos para o Neotrópico: Camposporium fusisporum Whitton, McKenzie \& Hyde; Cylindrocarpon curtum Bugnicourt; Minimidochium microsporum Matsush.; Sporidesmiella aspera Kuthub. \& Nawawi; Sporidesmium ghanaense M.B. Ellis. Stachybotrys theobromae Hansf. é citado pela primeira vez para o Brasil.

Palavras-chave: Diversidade, Fungos Anamorfos, Palmeiras, Amazônia

ABSTRACT - (Anamorphic Fungi (hyphomycetes) from Caxiuanã National Forest, Pará, Brazil. New records for the Neotropics). The hyphomycetes are important decomposers and recyclers of dead organic matter in the environment and may be important pathogens of plants and animals. As part of the diversity inventory of palm-tree fungi from Eastern Amazonia, in Caxiuanã National Forest, Pará, five new records of hyphomycetes for the Neotropics were found: Camposporium fusisporum Whitton, McKenzie \& Hyde; Cylindrocarpon curtum Bugnicourt; Minimidochium microsporum Matsush.; Sporidesmiella aspera Kuthub. \& Nawawi; Sporidesmium ghanaense M.B. Ellis. Stachybotrys theobromae Hansf. is cited for the first time for Brazil.

Key words: Diversity, Anamorphic Fungi, Palms, Amazonia

\section{Introdução}

Os hifomicetos estão incluídos entre os fungos anamorfos, sendo amplamente distribuídos no ambiente, presentes em diferentes habitats terrestres e aquáticos, e mesmo dispersos pelo ar na forma de conídios e/ou fragmentos de hifas (Kirk et al. 2008).

As palmeiras (Arecaceae) são importantes componentes dos ambientes naturais, devido a sua abundância e numerosas interações com outros organismos. Fornecem diversos produtos de grande interesse econômico a humanidade (Clement et al. 2005; Kahn \& De Granville 1992).

Os estudos sobre fungos associados à família Arecaceae se destacaram a partir do começo da década de 90 , e desde então, vêm revelando uma grande diversidade de fungos incluindo muitas espécies novas (Hyde et al. 2007).

No Brasil, poucos estudos de caráter taxonômico envolvendo fungos sobre palmeiras foram realizados, como os de Farr (1980) que descreveu a espécie Cryptophiale minor M.L Farr sobre folhas mortas da palmeira Astrocaryum sp. para o estado do Amazonas; Rodrigues (1994) realizou um estudo sobre fungos endofíticos presentes na palmeira Euterpe oleracea Mart. no estado do Pará; Grandi (1999) estudou os hifomicetos sobre folhedo em decomposição de Euterpe edulis Mart. no Estado de São Paulo; e para a Floresta Nacional de Caxiuanã, Pará, Gutiérrez et al. (2009) forneceram uma lista preliminar de hifomicetos presentes em palmeiras dos gêneros Astrocaryum sp., Bactris sp., Euterpe sp., Geonoma sp., Maximiliana sp. e Oenocarpus sp.

O presente trabalho tem como objetivo relatar os hifomicetos presentes em folhas de palmeiras que representam novos registros para o Neotrópico, ampliando a distribuição geográfica dessas espécies. Esse estudo faz parte do inventário de hifomicetos sobre palmeiras na Floresta Nacional de Caxiuanã (PA), que está sendo realizado no sítio do Programa de Pesquisa em Biodiversidade (PPBIO-Amazônia Oriental 2009).

\section{Material e métodos}

A Floresta Nacional de Caxiuanã ocupa uma área de aproximadamente 320.000 hectares nos municípios de Portel e Melgaço, no Pará, situada entre as coordenadas $01^{\circ} 37^{\prime} \mathrm{S} / 51^{\circ} 19^{\prime} \mathrm{W}$ e $01^{\circ} 54^{\prime} \mathrm{S} / 51^{\circ} 58^{\prime} \mathrm{W}$ e ao sul $02^{\circ} 15^{\prime} \mathrm{S} /$ $51^{\circ} 15^{\prime} \mathrm{W}$ e $02^{\circ} 15^{\prime} \mathrm{S} / 51^{\circ} 56^{\prime} \mathrm{W}$ (Montag et al. 2008). Em seu interior está implantado um sítio de estudo do PPBIO-Amazônia Oriental, tendo como referência as coordenadas $01^{\circ} 57^{\prime} 36^{\prime \prime} \mathrm{S}$ e $51^{\circ} 36^{\prime} 55^{\prime}$ W, com uma área de $25 \mathrm{Km}^{2}$ de floresta ombrófila densa, delimitada em forma de grade $(5 \mathrm{x}$ $5 \mathrm{Km}$ ) por um sistema de parcelas e trilhas (PPBIO-Amazônia Oriental 2009), onde foram efetuadas as coletas deste estudo.

Foram realizadas duas expedições à área de estudo (dezembro/2006 e agosto/2007), onde amostras compostas de partes de folhas de palmeiras (folíolos, ráquis e pecíolos) em decomposição de palmeiras foram coletadas. As partes coletadas foram imediatamente secas em estufa de campo a gás, desta forma, em função da metodologia adotada, somente as estruturas presentes no momento da coleta foram analisadas.

As folhas em decomposição foram observadas em estereomicroscópio e as estruturas fúngicas retiradas com auxílio de estiletes, para a preparação de lâminas semipermanentes montadas em lactoglicerol acrescidos de

\footnotetext{
1 Parte da dissertação de Mestrado da primeira Autora

2 Museu Paraense Emílio Goeldi, Coordenação de Botânica, Belém, PA, Brasil

3 Universidade Federal do Pará, Instituto de Ciências Biológicas, Belém, PA, Brasil

4 Autora para correspondência: helen@museu-goeldi.br
} 
azul de Amann, para posterior observação em microscópio óptico de luz, onde foram realizadas as medições das microestruturas de importância taxonômica. Literatura especializada do grupo foi utilizada, tais como: Ellis (1971, 1976), Matsushima (1980, 1993, 1996). Amostras com os espécimes estudados foram incorporadas ao Herbário João Murça Pires (MG) do Museu Paraense Emílio Goeldi, Belém (PA)

\section{Resultados e discussão}

$\mathrm{O}$ estudo dos fungos coletados revelou a presença de seis novos registros de hifomicetos para o Neotrópico, demonstrando a importância da realização de levantamentos em áreas pouco exploradas e da abordagem de grupos megadiversos, pouco estudados como os hifomicetos. A seguir apresentam-se os táxons identificados.

1. Camposporium fusisporum Whitton, McKenzie \& K. D. Hyde, Fungal Diversity 11: 180, 2002.

Descrição e ilustração: Whitton et al. (2002).

Material selecionado: BRASIL. Pará: Melgaço, FLONA de Caxiuanã, sobre folíolo e pecíolo em decomposição de Geonoma baculifera (Poith.) Kunth., 14/XII/2006, Hernández, A. s.n. (MG 190705).

Distribuição geográfica: Brunei, sudeste asiático (Whitton et al. 2002).

Comentários: Camposporium fusisporum caracteriza-se pelos conídios 8-11 septados, fusiformes, alongados, com 2-3 apêndices apicais asseptados. Whitton et al. (2002) revisaram o gênero Camposporium e elaboraram uma chave para 14 espécies aceitas, segundo esses autores $C$. laundonii assemelha-se a C. fusisporum pela morfologia dos conídios, diferenciando-se por apresentar geralmente um apêndice e quando produz dois, estes são septados e parecem extensões alongadas do conídio, enquanto C. fusisporum apresenta dois apêndices asseptados e divergentes. Os espécimes encontrados na FLONA Caxiuanã apresentaram conidióforos maiores (108-205 $\mu \mathrm{m})$, conídios menores $(75-92 \mu \mathrm{m})$, e apêndices maiores $(28-55 \mu \mathrm{m})$, que os citados na descrição original (100-145 $\mu \mathrm{m}$ (conidióforos),

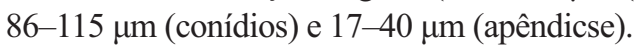

2. Cylindrocarpon curtum Bugnic., Encycl. Mycol. 11: 181, 1939.

Descrição e ilustração: Matsushima (1980).

Material selecionado: BRASIL. Pará: Melgaço, FLONA de Caxiuanã, sobre pecíolo em decomposição de Bactris hirta Mart., 17/VIII/2007, Monteiro, J.S. s.n. (MG 190752).

Distribuição geográfica: Taiwan e Vietnã (Matsushima 1980).

Comentários: O gênero Cylindrocarpon apresenta 135 espécies descritas (incluindo vários sinônimos) e possui como teleomorfos ascomicetos da família Nectriaceae (Kirk \& Cooper 2008). A espécie C. curtum possui conidióforos simples, que consistem de uma única fiálide e conídios 1-3 septados (geralmente 2-septos), cilíndricos, com extremidades arredondadas. Os espécimes analisados concordaram com a descrição de Matsushima (1980), o qual refere que esta espécie tem sido isolada com pouca frequência no ambiente.
3. Minimidochium microsporum Matsush., Mats. Mycol. Mem. 9: 16-17, 1996.

Descrição e ilustração: Matsushima (1996).

Material selecionado: BRASIL. Pará: Melgaço, FLONA de Caxiuanã, sobre folíolos em decomposição de Geonoma baculifera (Poith.) Kunth, 14/XII/2006, Hernández, A. s.n. (MG 190705).

Distribuição geográfica: Japão (Matsushima 1996).

Comentários: O gênero Minimidochium possui sete espécies descritas atualmente (Kirk \& Cooper 2008). Minimidochium microsporum possui conídios pequenos (3-5 $\mu \mathrm{m})$ quando comparados às demais espécies do gênero. Assemelhase a M. parvum Cab., Aramb. \& Cazau, porém esta apresenta conídios maiores $(8-12 \mu \mathrm{m})$. Os espécimes estudados concordaram com a descrição de Matsushima (1996).

4. Sporidesmiella aspera Kuthub. \& Nawawi, Mycol. Res. 97(11): 1305-1306, 1993.

Descrição e ilustração: Kuthubutheen \& Nawawi (1993).

Material selecionado: BRASIL. Pará: Melgaço, FLONA de Caxiuanã, sobre folíolo em decomposição de Bactris hirta Mart., 14/XII/2006, Hernández, A. s.n. (MG 190708).

Distribuição geográfica: Malásia (Kuthubutheen \& Nawawi 1993).

Comentários: O gênero Sporidesmiella apresenta 28 espécies descritas (Kirk \& Cooper 2008). Yanna et al. (2001) elaboraram uma chave para 22 espécies. Sporidesmiella aspera possui conidióforos verrucosos desde a metade até logo abaixo da zona conidiogênica; esta característica a separa das outras espécies desse gênero. Os espécimes analisados concordaram com a descrição original, mas apresentaram conidióforos maiores $(150-240 \mu \mathrm{m})$ e conídios menores (11-19 $\mu \mathrm{m}$ ) em relação ao do material-tipo (até $160 \mu \mathrm{m}$ e 17-22 $\mu \mathrm{m}$, respectivamente).

5. Sporidesmium ghanaense M. B. Ellis, Mycol. Pap. 70: 50-51, 1958.

Descrições e ilustrações: Ellis (1958, 1976).

Material selecionado: BRASIL. Pará: Melgaço, FLONA de Caxiuanã, sobre folíolos em decomposição de Astrocaryum gynacanthum Mart., 24/VIII/2007, Monteiro, J.S. s.n. (MG 190740).

Distribuição geográfica: Gana, Serra Leoa (Ellis 1976), Costa do Marfim (Rambelli et al. 2004).

Comentários: O gênero Sporidesmium possui mais de 400 espécies descritas, incluindo os sinônimos (Kirk \& Cooper 2008). A espécie possui conídios com 2-3 células medianas castanho-escuras e células apicais que se tornam gradualmente mais claras. Os espécimes analisados estão de acordo com a descrição de Ellis (1958), porém apresentaram conidióforos maiores $(66-150 \mu \mathrm{m})$ e conídios menores (24$37 \mu \mathrm{m}$ ) que os descritos para a espécie (até $130 \mu \mathrm{m}$ e 31-53 $\mu \mathrm{m}$, respectivamente). Sporidesmium ghanaense tem sido pouco isolada do ambiente, ocorrendo principalmente em regiões tropicais (Ellis 1976). 
6. Stachybotrys theobromae Hansf., Proc. Linn. Soc. Lond. 155: 45, 1943.

Descrições e ilustrações: Ellis (1971), Hansford (1943), Hughes (1952) e Matsushima (1993).

Material selecionado: BRASIL. Pará: Melgaço, FLONA de Caxiuanã, sobre folíolos em decomposição de Bactris hirta Mart., 17/VIII/2007, Monteiro, J.S. s.n. (MG 190738).

Distribuição geográfica: Costa do Marfim (Hughes 1952), Gana, Malásia, Nigéria, Papua-Nova Guiné, Serra Leoa, Trinidade (Ellis 1971), Sri Lanka (Matsushima 1993), Uganda (Hansford 1943).

Comentários: Esta espécie representa um novo registro para a FLONA Caxiuanã e para o Brasil. O gênero Stachybotrys possui 53 espécies descritas (Pinruan et al.2004) e seus teleomorfos estão relacionados à ordem Hypocreales (Kirk \& Cooper 2008). A espécie caracteriza-se pelos conídios elipsóides, negros, com um pequeno apículo projetado na base. Os espécimes analisados concordaram com as descrições de Hughes (1952), Ellis (1971) e Matsushima (1993). Stachybotrys theobromae já foi registrado em pecíolos de uma palmeira não identificada (Matsushima 1993).

\section{Agradecimentos}

Os autores agradecem ao PPBIO-Amazônia Oriental pelo financiamento e apoio logístico do projeto; ao MPEG e ao Laboratório de Micologia (UFPA) pela infraestrutura oferecida; à Dra. Rosely Ana Piccolo Grandi pela colaboração na identificação das espécies e bibliografia cedida; ao Luís Carlos Lobato pela identificação das palmeiras; aos revisores da Revista Acta Botânica pelas sugestões a este artigo; ao Conselho Nacional de Desenvolvimento Científico e Tecnológico $(\mathrm{CNPq})$ pela concessão da bolsa de mestrado ao primeiro autor.

\section{Referências bibliográfica}

Clement, C.R.; Lleras Pérez, E. \& Van Leeuwen, J. 2005. O potencial das palmeiras tropicais no Brasil: acertos e fracassos das últimas décadas. Agrociencias 9(1-2): 67-71.

Ellis, M.B. 1958. Clasterosporium and some allied dematiaceae phragmosporae. 1. Mycological Papers 70: 1-89.

Ellis, M.B. 1971. Dematiaceous Hyphomycetes. Kew, Commonwealth Mycological Institute.

Ellis, M.B. 1976. More Dematiaceous Hyphomycetes. Kew, Commonwealth Mycological Institute.

Farr, M.L. 1980. A new species of Cryptophiale from Amazonas. Mycotaxon 11: 177-181.
Grandi, R.A.P. 1999. Hifomicetos decompositores do folhedo de Euterpe edulis Mart. Hoehnea 26(1): 87-101.

Gutiérrez, A.H.; Monteiro, J.S. \& Sotão, H.M.P. 2009. Hifomicetos (fungos anamorfos) associados a palmeiras na Floresta Nacional de Caxiuanã, PA, Brasil. Pp. 561-563. In: Lisboa, P. (Org.). Caxiuanã: Desafios para a conservação de uma floresta nacional na Amazônia. Belém, Museu Paraense Emílio Goeldi.

Hansford, C.G. 1943. Contribution towards the fungus flora of Uganda. V. Fungi Imperfecti. Proceedings of the Linnean Society of London 155: 34-67.

Hyde, K.D.; Bussaban, B.; Paulus, B.; Crous, P.W.; Lee, S.; McKenzie, E.H.C.; Photita, W. \& Lumyong, S. 2007. Diversity of saprobic microfungi. Biodiversity and Conservation 16(1): 7-35.

Hughes, S.J. 1952. Fungi from the Gold Coast I. Mycological Papers 48: 1-91.

Kahn, F. \& De Granville, J.J. 1992. Palms in forest ecosystems of Amazonia. Ecological Studies 95. Berlin, Springer Verlag.

Kirk, P.M.; Cannon, P.F.; David, J.C. \& Stalpers, J.A. 2008. Ainsworth and Bisby's Dictionary of the Fungi. Wallingford, CAB International.

Kirk, P.M. \& Cooper, J. 2008. Index fungorum. http://www.indexfungorum. org (acesso em 13/09/2009).

Kuthubutheen, A.J. \& Nawawi, A. 1993. Three new and several interesting species of Sporidesmiella from submerged litter in Malaysia. Mycological Research 97(11): 1305-1314.

Matsushima, T. 1980. Saprophytic microfungi from Taiwan. Part 1. Hphomycetes. Matsushima Mycological Memoirs $\mathbf{N}^{0} 1$. Kobe, Publicado pelo autor.

Matsushima, T. 1993. Matsushima Mycological Memoirs No 7. Kobe, Publicado pelo autor.

Matsushima, T. 1996. Matsushima Mycological Memoirs No 9. Kobe, Publicado pelo autor.

Montag, L.F.A.; Freitas, T.M.S.; Wosiacki, W.B. \& Barthem, R.B. 2008. Os peixes da Floresta Nacional de Caxiuanã (municípios de Melgaço e Portel, Pará - Brasil). Boletim do Museu Paraense Emílio Goeldi Ciências Naturais 3(1): 11-34.

PPBIO Amazônia Oriental 2009. http://marte.museu-goeldi.br/ppbio/ ppbio15/ (acesso em 02/2009).

Pinruan, U.; McKenzie, E.H.C.; Jones, E.B.G. \& Hyde, K.D. 2004. Two new species of Stachybotrys, and key to the genus. Fungal Diversity 17: $145-157$.

Rambelli, A.; Mulas, B. \& Pasqualetti, M. 2004. Comparative studies of microfungi in tropical ecosystems in Ivory Coast forest litter: behaviour on different substrata. Mycological Research 108(3): 325-336.

Rodrigues, K.F. 1994. The foliar fungal endophytes of the Amazonian palm Euterpe oleracea. Mycologia 86(3): 376-385.

Whitton, S.R.; McKenzie, E.H.C. \& Hyde, K.D. 2002. Microfungi on the Pandanaceae: two new species of Camposporium and key to the genus. Fungal Diversity 11: 177-187.

Yanna, W.H.H.; Hyde, K.D. \& McKenzie, E.H.C. 2001. Sporidesmiella oraniopsis, a new species of dematiaceous hyphomycete from North Queensland, Australia and synopsis of the genus. Fungal Diversity 8: $183-190$. 